
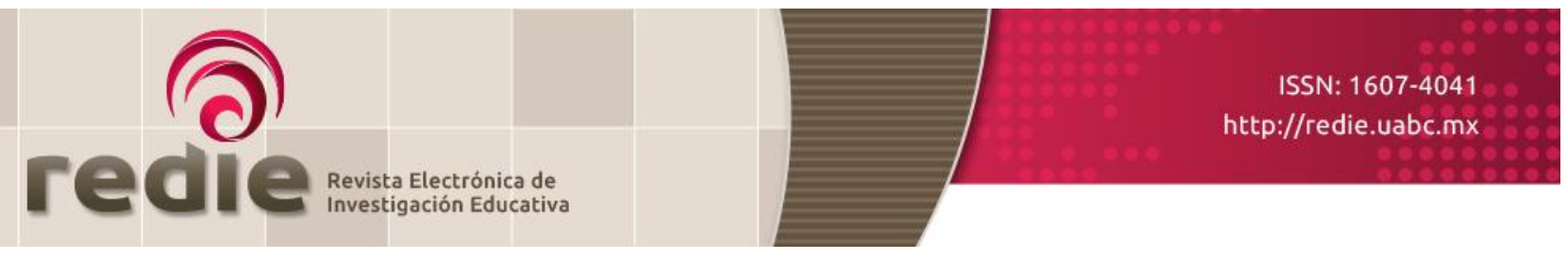

Vol. 22, 2020/e16

\title{
Diseño pedagógico adaptativo para el desarrollo de mooc: una estrategia para el desarrollo de competencias en contextos corporativos
}

\section{Adaptative Pedagogical Design for mooc Development: A Strategy for Developing Competences in Corporate Contexts}

Isabel Jiménez Becerra (*) https://orcid.org/0000-0003-4263-1970

Orlando Elias Fernández Palma (*) https://orcid.org.0000-0001-7862-1972

Fanny Teresa Almenárez Moreno (*) https://orcid.org.0000-0001-8165-3561

(*) Universidad de La Sabana

(Recibido: 13 de enero de 2018; Aceptado para su publicación: 26 de junio de 2018)

Cómo citar: Jiménez, I., Fernández, O. E. y Almenárez, F. T. (2020). Diseño pedagógico adaptativo para el desarrollo de mooc: una estrategia para el desarrollo de competencias en contextos corporativos. Revista Electrónica de Investigación Educativa, 22, e16, 118. https://doi.org/10.24320/redie.2020.22.e16.2192

\section{Resumen}

El artículo plantea los aportes de los cursos abiertos masivos en línea (MOOc, por sus siglas en inglés) a los procesos de aprendizaje corporativo, con base en un modelo pedagógico adaptativo. La investigación se desarrolló bajo un enfoque mixto en el marco de un diseño exploratorio de alcance explicativo, sustentado en variables como la enseñanza adaptativa y la técnica didáctica del Aprendizaje Basado en Problemas (ABP), así como los efectos en el aprendizaje significativo. Los resultados presentan algunas contribuciones de los MOOC a los procesos de gestión en las organizaciones y al desarrollo de destrezas tecnológicas, las cuales conducen al fortalecimiento y éxito de las organizaciones en el contexto corporativo.

Palabras clave: Aprendizaje adaptativo, Aprendizaje Basado en Problemas (ABP), MOoc, metodologías de aprendizaje.

\section{Abstract}

This article intends to present the contributions of online open mass courses (MOoc) to corporate learning processes, based on an adaptive pedagogical model. To this end, the research was developed under a mixed, within the framework of an exploratory design of explanatory scope, based on variables such as adaptive teaching and the didactic technique of Problem Based Learning (PBL), as well as effects on meaningful learning. The results present some contributions of MOOC to management processes in organizations and to the development of technological skills, which lead to the strengthening and success of organizations in the corporate context.

Keywords: Adaptive learning, Problem Based Learning (PBL), MOOc, learning methodologies. 


\section{Introducción}

En los sectores educativo y corporativo se presentan discusiones sobre estrategias para formar a sus empleados de manera exitosa, en el desarrollo de destrezas tecnológicas que cualifiquen los procesos de gestión organizacional. En este sentido, los MOOC -por sus características (masivos, gratuitos, interactivos y colaborativos) - se constituyen en elementos clave para llevar conocimientos y aprendizajes pertinentes y acordes al perfil de los participantes.

Diversas investigaciones, entre ellas Warnock (1978) y Marchesi et al. (2017), han demostrado que el éxito en este escenario de aprendizaje se fundamenta en un modelo pedagógico adaptativo; es decir, que contenga un método educativo en contexto con las necesidades de las empresas y, por tanto, con los participantes. De esta manera se genera la búsqueda de recursos de enseñanza interactiva acompañados de un proceso de evaluación que se enmarque no sólo en la certificación, si no en la evidencia de lo aprendido, tanto en la materialización de las actividades dentro del mooc como en el impacto generado en el contexto corporativo.

En la presente investigación se constituyó un modelo pedagógico adaptativo que se implementó en algunos MOoc para operacionalizar con funcionarios públicos de entidades estatales. El modelo concentró las siguientes dimensiones: metodología de Aprendizaje Basado en Problemas (ABP); caracterización de los participantes y concepción del conocimiento brindado; objetivos de aprendizaje, en concordancia con el proceso de gestión que se buscaba articular en las entidades; experiencias de aprendizaje mediante laboratorios de práctica y recursos variados; así como una interacción colaborativa basada en un diseño pedagógico e instruccional, en cuyas fases se aplicó el modelo pedagógico adaptativo.

Del mismo modo, en el año 2016 se desplegaron dos Mooc, cada uno con dos meses de duración, cuya convocatoria obtuvo 1300 inscritos y una deserción inferior a 60\%. Durante el proceso se generó una investigación de diseño correlacional para correlacionar variables sobre la enseñanza adaptativa basada en el enfoque de ABP y sus efectos en el aprendizaje significativo.

\subsection{El modelo pedagógico adaptativo de los MOOC}

La iniciativa de implementar los mooc en contextos institucionales con el objetivo de generar experiencias de aprendizaje adaptativas que apuntaran a cualificar los procesos de gestión organizacional en algunas entidades públicas colombianas -mediante el fortalecimiento de las destrezas tecnológicas aplicadas al contexto laboral- implicó analizar la esencia del modelo en situaciones concretas. Esto surgió en 2012 y soportó diferentes transformaciones hasta el año 2014, producto de las críticas originadas por la deserción de los participantes, cuya tasa promedio de finalización era inferior a 7\% (Рarr, 2013).

Otra crítica apuntaba a la carencia que se presenta en la relación educativa (Postic, 2000), al considerar que los MOOC no pueden reemplazar la valiosa interacción de los estudiantes con el profesor, ya que muchos pedagogos y expertos en didáctica consideran que es un elemento esencial para el proceso enseñanzaaprendizaje. Con base en esta postura, quienes implementan los mooc han buscado estrategias para mejorar las formas de interacción al plantear las experiencias de simulación con laboratorios, buscando fortalecer los procesos de socialización y colaboración entre los participantes (Bates, 2012).

A pesar de sus desventajas, varios defensores de los mooc han destacado las ventajas que los han mantenido vigentes en el mundo académico e institucional. Sus bondades se enfocan en los procesos de aprendizaje generados desde los principios de conectivismo, interacción y colaboración que vivencian los participantes al socializar saberes y construir aprendizajes, basados en un modelo pedagógico exitoso (UNESCO, 2013). Por consiguiente, el modelo pedagógico debe sustentarse en un enfoque o metodología que respete la caracterización de los participantes mediante experiencias de aprendizaje basadas en situaciones de su contexto real, capaces de desarrollar sinergias en su implementación. Del mismo modo, esto debe acompañarse de objetivos de aprendizaje que respondan al modelo y a un proceso de autoevaluación formativo (Díaz, 2005). 
Los Mooc de Coursera se constituyen en un caso de éxito global no sólo por su diseño didáctico y pedagógico sino por el modelo de negocios que ofrece a las universidades estadounidenses (6 a 15\% de los ingresos brutos generados por cada mooc en su plataforma). Su prestigio se debe también al $20 \%$ de las ganacias generadas por el conjunto de cursos que suministran las universidades, materializa dos en las posibilidades que ofrece para complementar o reforzar los procesos de aprendizaje de los estudiantes (Kolowich, 2013a).

Otro elemento que robustece el modelo consiste en los recursos y las experiencias de interacción y colaboración que lo integran; por ejemplo, videos temáticos, programas de televisión, tutoriales, chat, foros, wikis, juegos, tests, experiencias de simulación o laboratorios, grupos de discusión sobre los temas en cuestión por medio de tutorización de terceros, apoyados por el conectivismo en redes sociales o blogs. El resultado de cada una de estas experiencias es la oportunidad de operacionalizar el modelo pedagógico, con base en su metodología, para favorecer las destrezas útiles y procesos de aprendizaje, lo cual puede ser implementado en un contexto productivo, académico u organizacional (Hollands y Tirthali, 2014).

En esta investigación, los MOoc no se implementaron de acuerdo con el paradigma de sustituir la educación presencial (Cann, 2013). En esencia, se articuló y ajustó mediante un modelo pedagógico adaptativo, planteado por los investigadores, como estrategia para generar acceso a toda la población participante en esta experiencia, así como en consolidar sus procesos de aprendizaje en el campo de las destrezas tecnológicas con impacto en la gestión organizacional.

Al retomar el modelo pedagógico adaptativo se debe ahondar en su conceptualización. Esto obedece a que el aprendizaje, para que sea exitoso, debe tener diferentes alternativas, acercamientos a un tema, organización del contenido, explicaciones, representaciones y ejemplos, así como material variado para que los estudiantes puedan elegir, con el propósito de hacer contacto con sus fortalezas e intereses (Snow,1997). En este sentido, lo que se busca es "incorporar diversas estrategias que permitan una actuación educativa global, coordinada y flexible, con el objetivo de conseguir que funcionen orquestadamente opciones alternativas de aprendizaje, en función de las características de cada alumno" (Fernández, 1993, p. 129).

En este orden de ideas, la metodología debe tener un perfil individual sobre el que se ajuste la instrucción, con base en sus debilidades y fortalezas, teniendo claro el tipo de objetivo o tema por trabajar, pues los resultados sólo tienen validez en un contexto limitado y definido (Snow, 1997). La principal dificultad que presenta este modelo es su aplicación en grupos amplios de personas, por tanto, es difícil elaborar estrategias individuales que le permitan a un docente dedicar tiempo suficiente a cada estudiante.

Por lo anterior, es una condición sine qua non que el docente se forme en competencias digitales, vistas éstas como el fortalecimiento de las habilidades y competencias de dichos actores para el uso eficaz de estos recursos en sus prácticas pedagógicas. En esta investigación se demostró que con una metodología articuladora y robusta un alto porcentaje de usuarios logró obtener procesos exitosos de aprendizaje.

\subsection{Componentes del modelo}

Los MOoc deben afrontar varios problemas para lograr experiencias de aprendizaje efectivas y evitar la deserción (Gaebel, 2014). Dentro de los principales problemas se destacan: el diseño pedagógico, la gestión económica ("monetización"), la certificación de los estudios ofrecidos, el seguimiento de la formación, la autentificación de los estudiantes y superar un modelo pedagógico encapsulado en un "e-learning empobrecido"; así como"la posibilidad de avanzar hacia modelos más colaborativos y competenciales, teniendo en cuenta la diversidad cultural y lingüística de diferentes zonas y contextos socioculturales" (Esteban y Eloy, 2014, p. 10).

Lo anterior constituye uno de los retos pedagógicos más grandes que enfrentan los diseñadores pedagógicos e instruccionales y los expertos temáticos, para crear mooc que mantengan la atención y, por consiguiente, la motivación por el aprendizaje entre los usuarios. Por esto, los investigadores plantearon un modelo pedagógico adaptativo que logró éxito por sus características de interacción, experiencias de 
aprendizaje colaborativo y baja deserción (inferior a 60\%), con base en sus componentes metodológicos. Con el propósito de describir el modelo se presentan sus dimensiones.

Diseño pedagógico e instruccional. El ABP combina técnicas de aprendizaje situado con técnicas que integran la gamificación y otros programas de simulación, que pueden relacionar a estudiantes y profesores de diversos lugares. En el caso de este MOOC, como ilustra en la figura 1, se articularon cinco fases con rutas de aprendizaje que permitieron operacionalizar el desarrollo didáctico, dado que orientaron los diferentes momentos de aprendizaje que podían abordarse en cualquiera de sus fases.

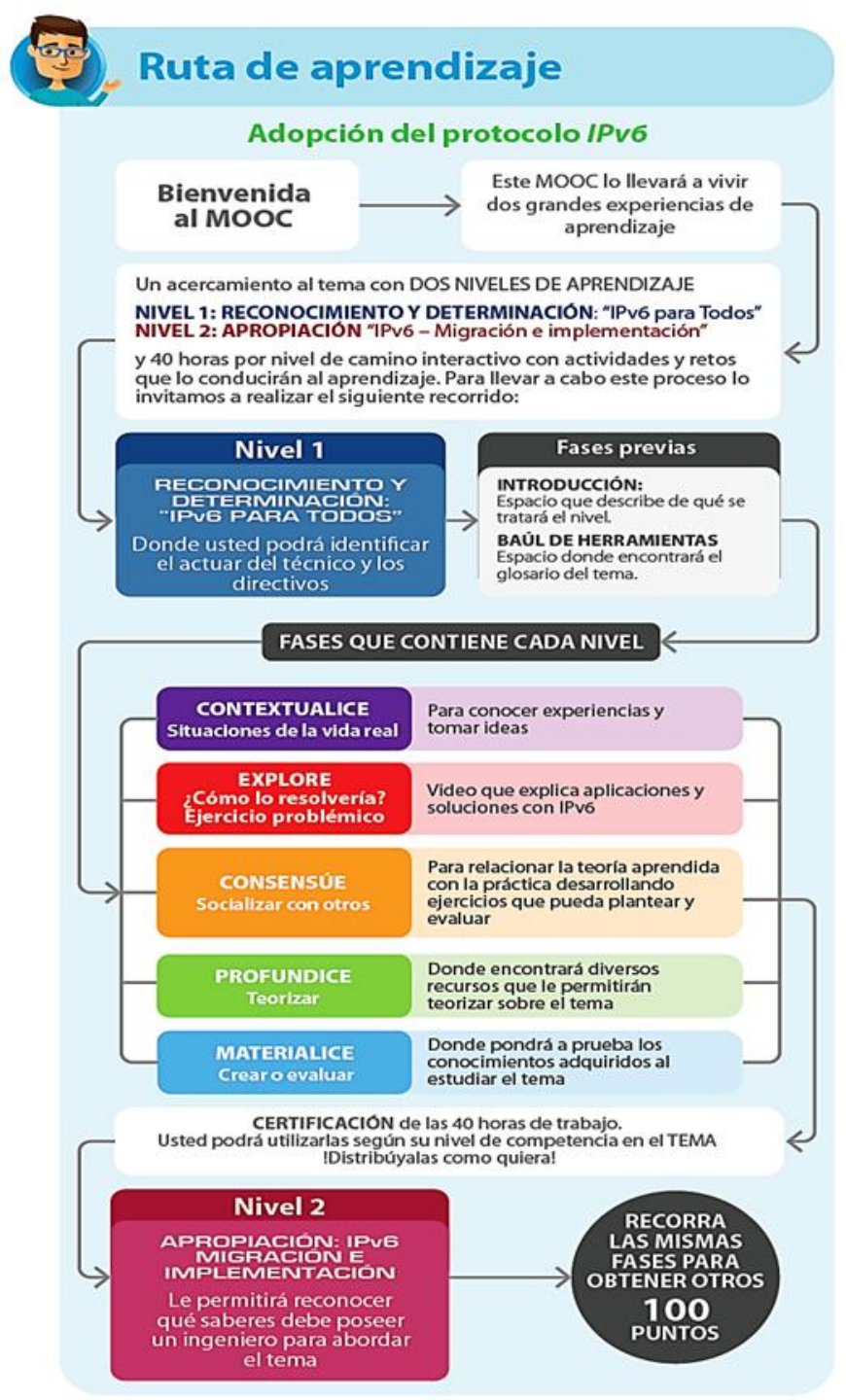

Figura 1. Ruta de aprendizaje del curso

Recursos. El origen de los recursos educativos digitales tiene dos fuentes: la desarrollada por el equipo de e-learning de la Red Nacioalna Académica de Tecnología Avanzada (RENATA), con base en el diseño instruccional y pedagógico, así como los recursos libres que se seleccionaron de Internet. A este respecto, el porcentaje de participación se distribuyó de la siguiente manera: RENATA desarrolló 68\% de los recursos y $32 \%$ correspondió a recursos libres, clasificados en animaciones, videos, presentaciones Prezi y PowerPoint, infografías, glosarios y calculadora virtual. 
Tipo de aprendizaje "adaptativo". Los agentes analizan el perfil del estudiante y logran adaptar el curso de diferentes maneras, a saber: ajustar el contenido del curso conforme al perfil y bagaje educativo de los participantes, de acuerdo con su localización o país de origen; por ejemplo, adecuando la lengua, las unidades de medida, los símbolos de la moneda y estaciones del año. También mostrando estudios de caso relevantes o lecturas complementarias según el país o región de origen, así como los intereses particulares (Daradoumis et al., 2013; Edwards, 2014). En el caso de estos Mooc, todos los usuarios, sin importar el perfil, podrían acceder a los diferentes niveles del mooc a partir de la adaptación de los contenidos por aprender, y a la aplicación de los conceptos en contextos y casuísticas.

Tipología del curso. En este apartado se presentan los tipos de curso articulados en el modelo mooc, así como la población que podría participar de este tipo de aprendizaje, el tiempo que se debe emplear y el proceso de aprendizaje que se enfrenta; estos elementos se especifican en la tabla I.

Tabla I. Tipología del curso

\begin{tabular}{|c|c|}
\hline \multicolumn{2}{|c|}{ MOoc adopción del protocolo ipv6 } \\
\hline Tipo de curso & $\begin{array}{l}\text { Modelo Mooc en Moodle con intención LMS (Learning } \\
\text { Management System) }\end{array}$ \\
\hline Público objetivo & $\begin{array}{l}\text { Dirigido a funcionarios públicos de las entidades del orden } \\
\text { nacional y territorial } \\
\text { Tácticos (técnicos), directivos e ingenieros }\end{array}$ \\
\hline & $\begin{array}{l}\text { Incluyente y abierto, con base en las capacidades y } \\
\text { niveles de reto de los participantes }\end{array}$ \\
\hline Modalidad & Virtual \\
\hline $\begin{array}{l}\text { Dos niveles } \\
\text { independientes }\end{array}$ & $\begin{array}{l}\text { Dos meses de trabajo } \\
40 \text { horas por nivel } \\
80 \text { horas en total } \\
\text { Nivel 1: "Reconocimiento y determinación" para } \\
\text { tácticos o técnicos a nivel operativo y para directivos } \\
\text { - Nivel 2: "Apropiación" para ingenieros }\end{array}$ \\
\hline Proceso de aprendizaje & Trabajo independiente respuesta automática \\
\hline
\end{tabular}

Objetivos de aprendizaje y competencias por desarrollar. Frente a este aspecto, cada nivel se articuló con la metodología de aprender haciendo, a fin de promover el aprendizaje social y colaborativo mediante la socialización de saberes y experiencias administrativas. Así, se propuso desarrollar habilidades cognitivas, estrategias y contextos al aplicar estos saberes en contexto. En la tabla II se describen sus elementos. 
Tabla II. Metodología del curso

\begin{tabular}{l|l}
\hline \multicolumn{2}{l}{ Adopción del protocolo IPv6 } \\
\hline Objetivo & $\begin{array}{l}\text { Generar proceso de aprendizaje con el fin de reconocer conceptos, características, } \\
\text { funcionalidades y principales aspectos básicos de sguridad con IPv6 y los mecanismos de } \\
\text { transición desde el IPv4. }\end{array}$ \\
\hline $\begin{array}{l}\text { Enfoque } \\
\text { pedagógico }\end{array}$ & Autoaprendizaje y aprendizaje social. \\
\hline $\begin{array}{l}\text { Enfoque } \\
\text { metodológico }\end{array}$ & $\begin{array}{l}\text { El enfoque metodológico se centra en la implementación de aprender haciendo. Los } \\
\text { aspectos teóricos cobran sentido si hay oportunidades para practicar lo aprendido } \\
\text { (Adaptado del programa Computadores para educar, MinTIC, p. 26) }\end{array}$ \\
\hline \multirow{2}{*}{$\begin{array}{l}\text { Eje de trabajo } \\
\text { articulador }\end{array}$} & $\begin{array}{l}\text { Las situaciones de la vida real constituyen la base significativa para el aprendizaje, pues se } \\
\text { busca partir de contextos concretos de los grupos objetivo. Esta estrategia permite la } \\
\text { inclusión y la movilidad de los actores del aprendizaje que en cada fase van generando } \\
\text { niveles de complejidad a partir de las funciones cotidianas del operativo, la intervención del } \\
\text { directivo y la disciplina del ingeniero. }\end{array}$ \\
\hline $\begin{array}{l}\text { Competencia tecnológica: selecciona y utiliza de forma pertinente, responsable y eficiente } \\
\text { una variedad de herramientas tecnológicas entendiendo los principios que las rigen, la } \\
\text { forma de combinarlas y las licencias que las amparan. } \\
\text { por desarrollar }\end{array}$ & $\begin{array}{l}\text { Competencia de gestión: utiliza el IPv6 en la planeación, organización, administración y } \\
\text { evaluación efectiva de los procesos administrativos o de programación en sus contextos. } \\
\text { Competencia investigativa: utiliza, analiza y está consciente de la importancia de aprender y } \\
\text { aplicar conocimientos sobre IPv6 para la transformación de la labor administrativa y la } \\
\text { generación de nuevos procesos (Adaptado del programa Computadores para educar, } \\
\text { MinTIC, p. 19) }\end{array}$ \\
\hline
\end{tabular}

Fuente: Elaboración propia a partir de Mintic (2015a).

Evaluación. Para relacionar el seguimiento y la monitorización del estudiante en la plataforma mooc es posible emplear tecnologías de aprendizaje automatizado. Al respecto, Universities United Kingdom (2013) proporciona un interesante resumen de estas herramientas emergentes, entre los que destacan: analítica web, tecnología de la web semántica y resolución virtual de problemas.

Teniendo en cuenta que los mooc se articulan con el aprender haciendo y el autoaprendizaje, en tanto formas metodológicas, la intención es generar una motivación permanente para que se reflejen materializaciones exitosas mediante las TIC, a fin de abordar los procesos de enseñanza-aprendizaje. En esa línea, el proceso evaluativo se concentra en la gamificación (tabla III) a fin de ofrecer las rúbricas de evaluación que debe llevar a la práctica el tutor del mooc. Se muestra una estructura general por procesos, porcentajes, niveles y fases; además, por horas de trabajo, puntos y valoraciones. 
Tabla III. Gamificación: sistema de valoración

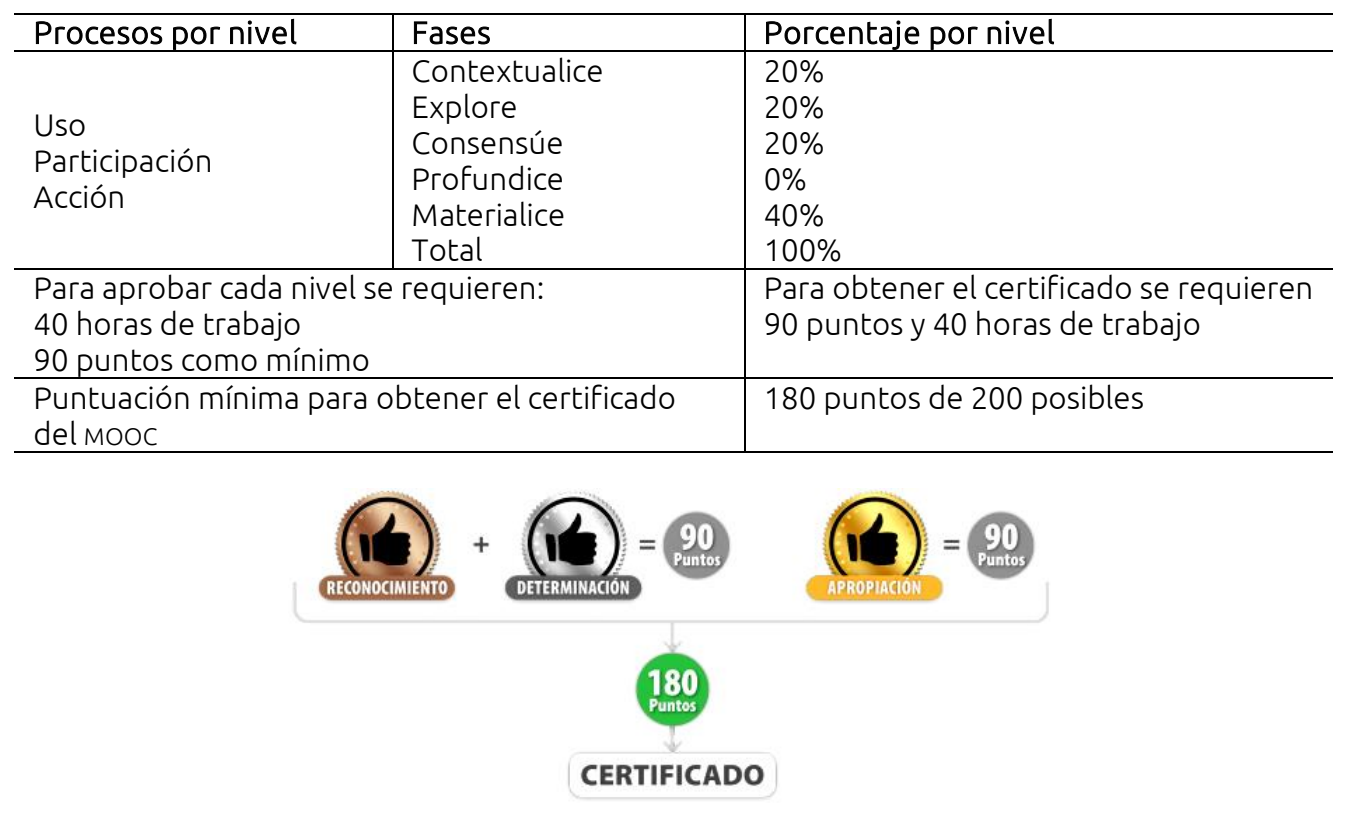

Fuente: Elaboración propia a partir de Mintıc (2015a).

Certificación. Al ser adaptativo, se avalaron todas las acciones de los participantes clasificadas en "participación" y "aprobación", siendo la idea central que se logre el aprendizaje a partir del recorrido del usuario por el mooc. Por esta razón se generaron las siguientes escalas de evaluación frente a su acción (ver tabla IV).

Tabla IV. Tipo de certificación según puntaje obtenido

\begin{tabular}{l|l}
\hline Certificación & Puntaje obtenido \\
\hline $\begin{array}{l}\text { Certificado } \\
\text { por participación }\end{array}$ & $\begin{array}{l}\text { Para aquellos que obtengan un puntaje mínimo de } 50 \text { puntos en un nivel, debido a } \\
\text { que no hicieron el entregable final en la fase "Materialice". }\end{array}$ \\
\hline $\begin{array}{l}\text { Certificado } \\
\text { de aprobación de un nivel }\end{array}$ & $\begin{array}{l}\text { Para aquellos participantes que obtengan } 90 \text { puntos por nivel y hayan realizado } \\
\text { todas las actividades del curso, materializadas en el entregable final. Este certificado } \\
\text { será generado por la entidad administradora, previa validación de los requisitos de } \\
\text { aprobación. }\end{array}$ \\
\hline $\begin{array}{l}\text { Certificado } \\
\text { de aprobación del mooc }\end{array}$ & $\begin{array}{l}\text { Para aquellos participantes que obtengan } 90 \text { puntos por nivel y un acumulado de } \\
\text { entidad administradora realizará la validación de los requisitos de aprobación antes } \\
\text { de la expedición de éste. }\end{array}$ \\
\hline
\end{tabular}

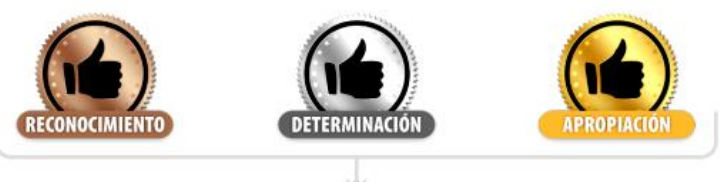

CERTIFICADO

Fuente: Elaboración propia a partir de Mintıc (2015a). 


\section{Método}

El ejercicio práctico de esta investigación se centró en difundir y posicionar los Mooc adaptativos. Esto es, "Adopción del protocolo IPv6" y "Mejores prácticas en la gestión y seguridad de TI", con el fin de propiciar la participación de los funcionarios responsables de las TIC en las entidades del Estado; en consonancia con el propósito del plan de comunicaciones de la Red Nacional de Investigación y Educación de Colombia (RENATA).

La investigación se desarrolló bajo un enfoque mixto en el marco de un diseño exploratorio de alcance explicativo, a partir de variables como la enseñanza adaptativa, cuya base fue el enfoque del ABP y sus efectos en el aprendizaje significativo, en el área de gestión pública. De acuerdo con Rojas (1989), este tipo de estudios pretende:

Analizar y explicar las relaciones existentes entre diferentes y múltiples variables y fenómenos sociales. Por lo tanto, mediante la elaboración y realización adecuada de instrumentos un investigador social puede llegar a responder preguntas sobre el qué, el cuándo, el cómo, el quién y también el porqué de la realidad en la que se ve inserto. (p. 178)

En la investigación participaron 1302 usuarios en el mooc "Adopción del protocolo IPv6" y 1325 usuarios en el MOOC "Mejores prácticas en la gestión y seguridad de TI" con certificación de 60\% de los inscritos en cada MOOc, para un total de 1574 usuarios certificados. La tabla $V$ resume las características de enfoque, diseño, técnicas e instrumentos usados y que se embebieron en las diversas actividades evaluadas dentro de cada MOOc. 
Tabla V. Enfoque, diseño, técnicas e instrumentos de las actividades evaluadas

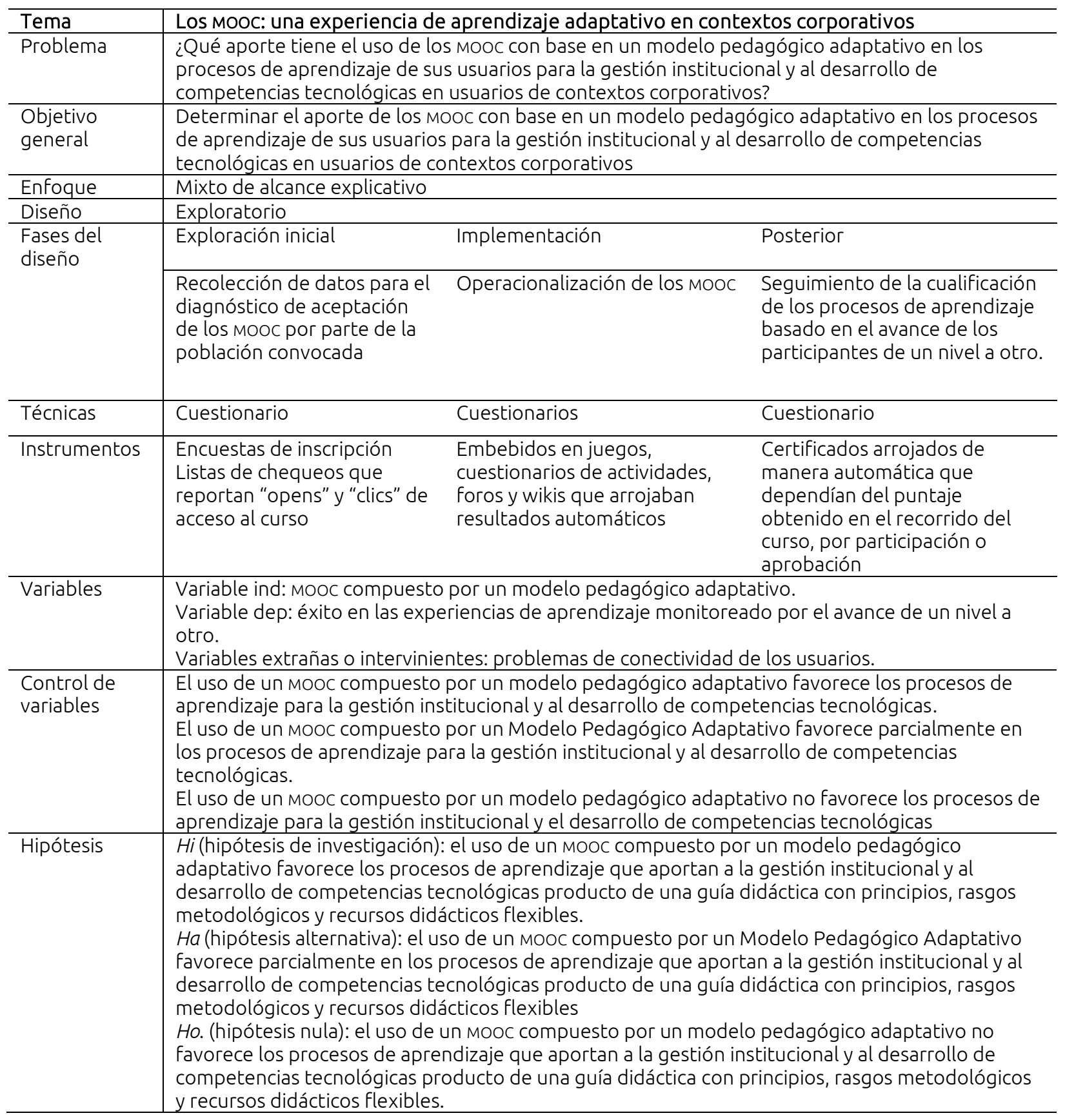

Fuente: Elaboración propia.

\section{Resultados}

Los resultados se presentan con base en las fases del diseño exploratorio seleccionadas para esta investigación y la explicación frente a sus alcances.

\subsection{Fase inicial. Recolección de datos para el diagnóstico de aceptación de los MOoC}

La fase inicial permitió identificar el contexto, la población y los temas por trabajar en el ambiente 
corporativo público. Luego del desarrollo de los MOoc, que contó con la participación de expertos temáticos, diseñadores pedagógicos, diseñadores gráficos, programadores de plataforma y líderes de proyectos, se materializaron los cursos y se inició la difusión.

Los cursos se monitorearon a partir de una campaña de expectativa dirigida a los funcionarios públicos; además, se generaron nueve campañas de seguimiento y reportes semanales que permitieron evidenciar el aumento en el número de usuarios y el uso constante de los Mooc.

En la figura 2 se muestra el reporte de seguimiento de los estudiantes de las instrucciones enviadas el 14 de octubre. En cada campaña se detallan la fecha de envío, los estudiantes a quienes se les envió, el porcentaje que leyó el correo y la cantidad de clics que hicieron en el botón "Ingreso a la plataforma Mooc", disponible en el mensaje enviado.

\begin{tabular}{|c|c|c|c|}
\hline Tercer Recordatorio Ingreso al MOOC IPv6 & 104 & $64.4 \%$ & $30.8 \%$ \\
\hline Regular - Inscritos MOOC IPv6 (segment) & Subscribers & Opens & Clicks \\
\hline \multicolumn{4}{|l|}{ Sent on Mon, Oct 25, 2015 10:33 am } \\
\hline Segundo envio instrucciones de ingreso a MOOC & 322 & $43.2 \%$ & $25.5 \%$ \\
\hline Regular - Inscritos MOOC IPv6 (segment) & Subscribers & Opens & Clicks \\
\hline \multicolumn{4}{|l|}{ Sent on Thu, Oct 22, 2015 08:23 pm } \\
\hline Inscritos MOOC adopción del protoclolo IFv6 & 1,302 & $77.9 \%$ & $63.1 \%$ \\
\hline Regular - Inscritos MOOC IPv6 (segment) & Subscribers & Opens: & Clicks \\
\hline Sent on Wed, Oct 14, 2015 01:43 pm & & & \\
\hline
\end{tabular}

Fuente: Mintıc (2015a).

Figura 2. Modelo y componentes a partir de las instrucciones enviadas a los participantes del mooc

Los resultados de uso evidencian la permanencia de los usuarios y la función del modelo pedagógico en el curso, materializado en una guía didáctica que integra los principios y rasgos metodológicos establecidos cuidadosamente dentro del mismo. Esto condujo a la flexibilidad y adaptabilidad, lo cual influyó para que el grupo de participantes se mantuviera en 60\% logrando avanzar en las tres fases del Mooc: Reconocimiento, Determinación y Apropiación. De manera que se cumplieron con todas las actividades de aprendizaje propuestas, pues para lograr los 180 puntos planteados en la gamificación de este artículo, los participantes debían concretar y ser evaluados por el sistema para poder avanzar.

\subsection{Fase implementación. Operacionalización de las Mooc}

En la segunda fase la investigación centró su ejercicio en la implementación y seguimiento de los cursos, a partir de la interacción y concreción por parte de los participantes, donde se revisó la ejecución de las actividades y el avance a través de las diferentes fases metodológicas del mooc. Las estadísticas descriptivas evidencian los resultados de este monitoreo. Para elaborar el informe se clasificaron los datos en cinco categorías que se recolectaron desde los formularios de inscripción, a saber:

- Clasificación por institucionalidad de los inscritos.

- Inscritos por género.

- Inscritos por departamento.

- Inscritos por nivel de estudios. 
Diseño pedagógico adaptativo para el desarrollo de Mooc: una estrategia para el desarrollo de competencias en contextos corporativos Jiménez et al.

- Inscritos por tipo de institución. 
La base de participantes para analizar y graficar la información está conformada por 1861 inscritos, con fecha de corte al 31 de diciembre de 2015. De 1861 inscritos, 27 han registrado correos electrónicos que no existen y 164 se inscribieron más de una vez con el mismo correo electrónico. Adicionalmente, se descartaron 96 inscritos pertenecientes al sector privado, por lo que se completó un total de 1574 inscritos.

Clasificación por institucionalidad de los inscritos. Los inscritos se clasificaron en: Gobierno, Gobierno educativo, Educativo privado y ONG (Organización No Gubernamental). En la figura 3 se ilustra la participación de los inscritos, donde se observa que 1308 participantes (83\%) corresponden a entidades adscritas al Gobierno; 182 (11.6\%) corresponden a entidades educativas adscritas al Gobierno; 78 (5\%) corresponden a entidades educativas de carácter privado y sólo 6 participantes (0.4\%) a las ONG.

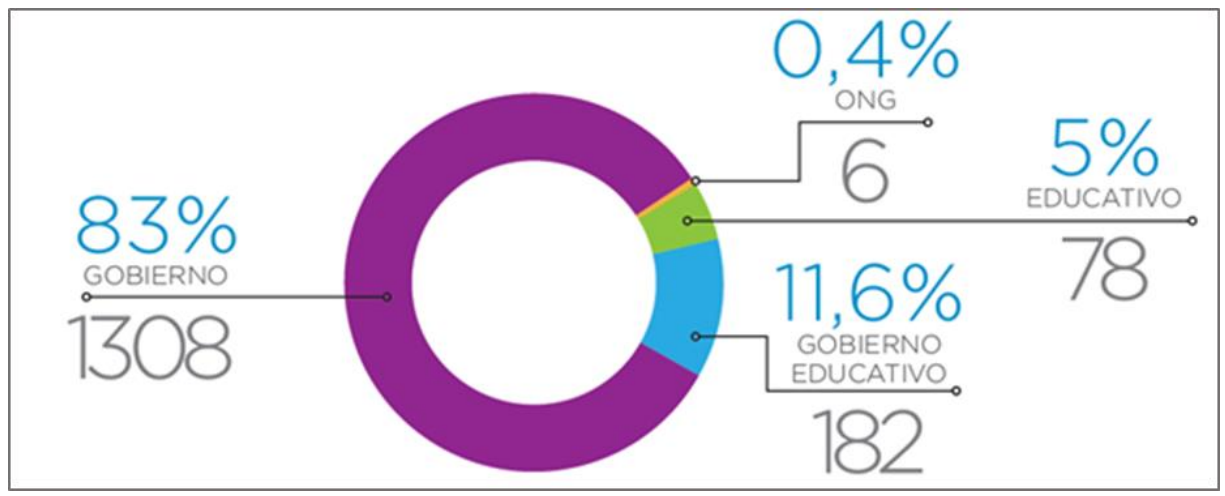

Fuente: Mintıc (2015b).

Figura 3. Clasificación de los inscritos por su institucionalidad

Funcionarios inscritos por género. En la figura 4 se presenta la participación por género de los funcionarios inscritos. En ésta se evidencia que el mooc es más llamativo para el género masculino, con 1 287 inscritos (82\% de participación); mientras del género femenino hubo 287 inscritas (18\% de participación). Esto puede obedecer al perfil de quienes desempeñan funciones relacionadas con el mooc.

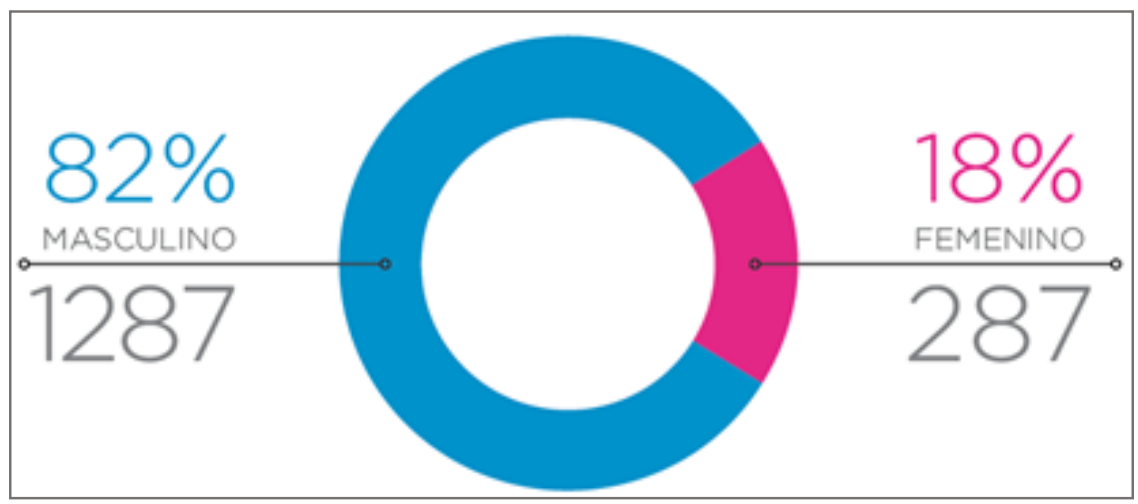

Fuente: MinTic (2015b).

Figura 4. Funcionarios inscritos por género

Funcionarios inscritos por departamento. En la figura 5 se detalla el porcentaje de inscritos por departamento. Cabe aclarar que Bogotá, por su importancia y población, se diferencia de Cundinamarca con una participación de 38\% o 604 inscritos y, tal como se observa, tiene más funcionarios inscritos que los otros departamentos juntos, con 18\% o 287 funcionarios inscritos. Es decir, 56\% de los funcionarios trabaja en Valle, Antioquia, Atlántico y Bogotá. Esto muestra que las necesidades de formación en tecnología están concentradas en estas regiones del país. 


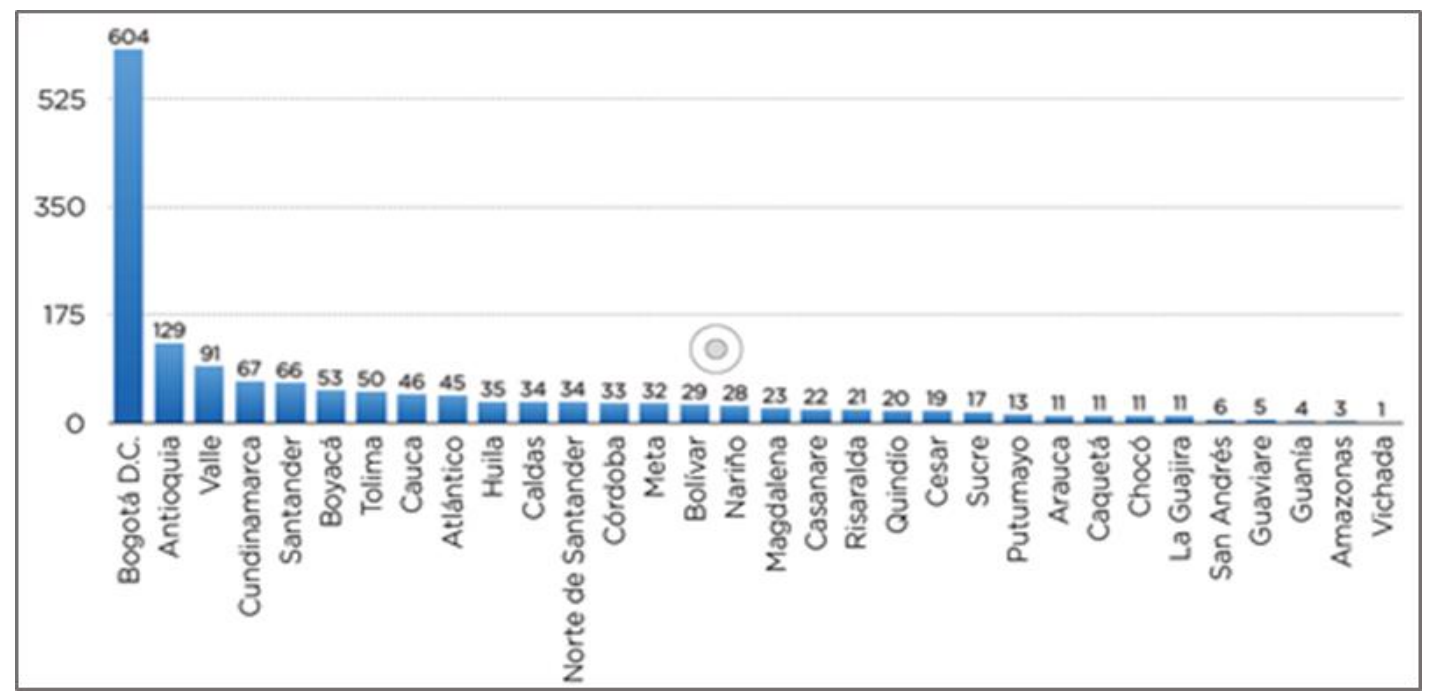

Fuente: Mintic (2015b).

Figura 5. Funcionarios inscritos por departamento

Funcionarios inscritos por nivel de estudios. La figura 6 presenta el nivel de estudios de los funcionarios inscritos. Se destacan quienes tienen formación técnica, con una participación de 36\% o 580 inscritos; seguidos de 26\% o 420 inscritos con estudios de pregrado, y 20\% o 327 inscritos con estudios de especialización; por otra parte, 0.3\% (5 inscritos) realizaron estudios de doctorado. Finalmente, 8\% (137 inscritos) son bachilleres, mientras que 6.6\% (105 inscritos) cuentan con estudios de maestría.

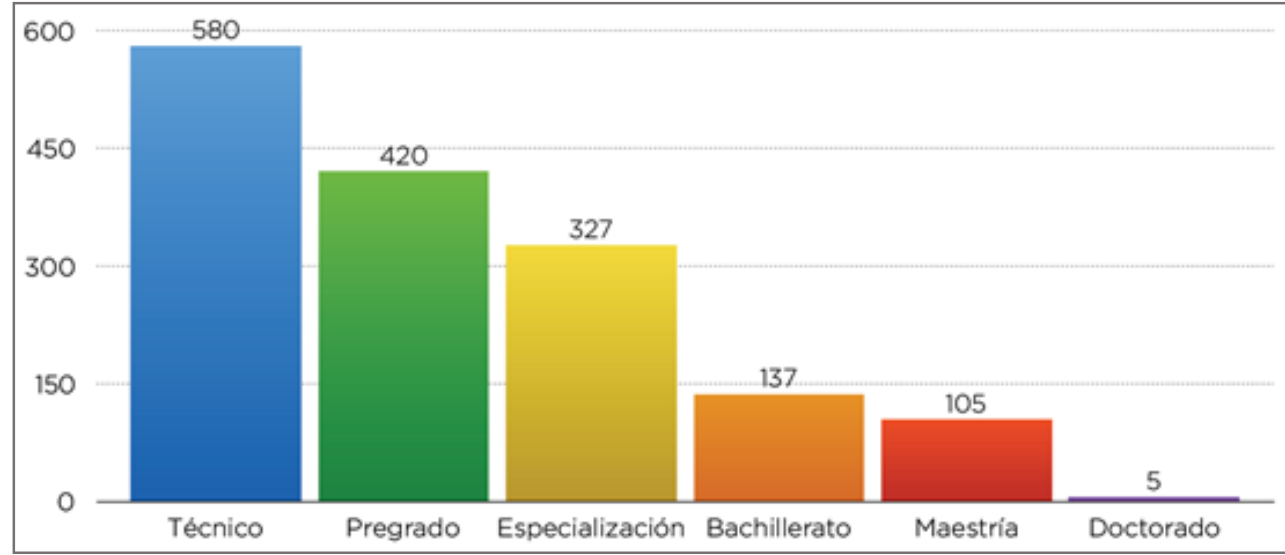

Fuente: Mintic (2015b).

Figura 6. Funcionarios inscritos por nivel de estudios 
Funcionarios inscritos por tipo de institución. En la figura 7 se ilustra la participación de los inscritos de acuerdo con el tipo de institución a la que están vinculados. En este caso, 86\% (1 357 inscritos) están vinculados a entidades de orden nacional, mientras que el 14\% (217 inscritos) son funcionarios de entidades de orden territorial.

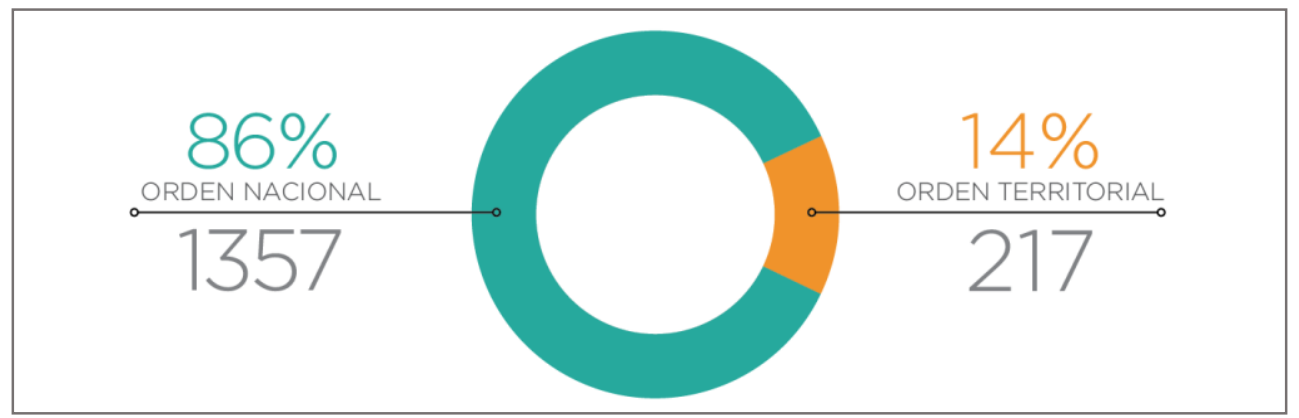

Fuente: Mintic (2015b).

Figura 7. Funcionarios inscritos por tipo de institución

\subsection{Fase posterior. Seguimiento de uso y cualificación de los procesos de aprendizaje}

En la fase final se identificó la cantidad de participantes que finalizó el curso y se certificó. Esto permitió evidenciar que la deserción no superó el $60 \%$ del total de los usuarios. Otro elemento que reflejan los resultados fue el avance en los procesos de aprendizaje, a partir de la culminación exitosa de cada actividad de un nivel a otro, producto de las fases de aprendizaje que posee el curso y el propósito de cada una. Algunos de los datos esenciales se generan en las certificaciones, como puede observarse a continuación.

Estudiantes certificados. Los estudiantes inscritos al mooc "Adopción de mejores prácticas en la gestión y seguridad de TI" fueron 1 574. En la figura 8 se ilustra el número de estudiantes certificados por nivel, así como el resultado de aquellos participantes que culminaron y se certificaron en los tres niveles (233 estudiantes).

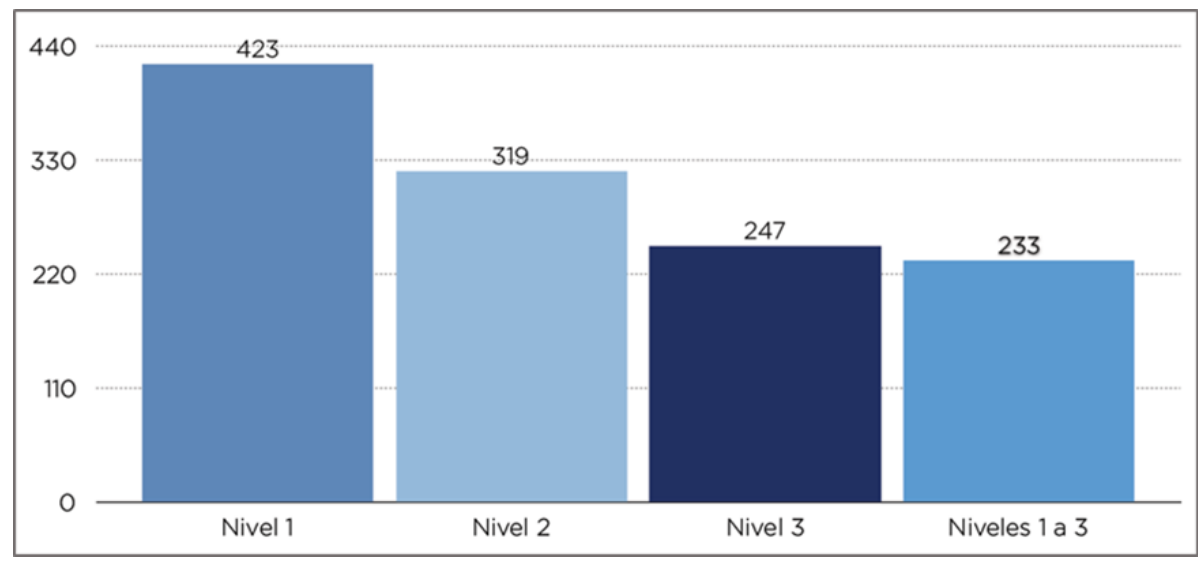

Fuente: Mintic (2015b).

Figura 8. Estudiantes certificados

\section{Discusión}

Dentro del análisis de resultados extraídos en las fases de diseño se respondió al objetivo general de la investigación, que era determinar el aporte de los mooc con base en un modelo pedagógico adaptativo en los procesos de aprendizaje de sus usuarios para la gestión institucional y al desarrollo de competencias tecnológicas en usuarios de contextos corporativos. Esto se evidencia en la fase posterior del diseño, al 
mostrar que sólo 40\% de los participantes del curso no logró la certificación. Sin duda, es una muestra de que la certificación no es un elemento determinante; de ahí que, sea posible confirmar que la mayoría de los estudiantes que recurren a un mooc ya poseen un título (Liyanagunawardena et al., 2013).

Otra aspecto analizado en la investigación se fundamenta en las características del modelo pedagógico adaptativo, que permitió a los participantes, sin importar el nivel de formación, aplicar la resolución de problemas a las casuísticas construidas por los expertos temáticos y diseñadores pedagógicos, en el marco de los contextos de gestión laboral aplicables a su realidad. Lo anterior responde a principios esenciales de los MOOC, que se adaptan culturalmente para garantizar la inclusión de todos los participantes tanto en debates como en foros, así como al evitar contribuciones culturalmente inaceptables (Mak et al., 2010).

Por otro lado, es de considerar que la Corporación RENATA financió esta investigación con el objetivo de implementar los MOoc en el contexto laboral de los funcionarios públicos en función de sus competencias y destrezas. Esto responde a iniciativas como la desarrollada por el gobierno de Tanzania -cuyo proyecto piloto, apoyado por el Banco Mundial, tuvo como propósito ofrecer cursos de la plataforma Coursera para ayudar a los estudiantes a adquirir destrezas tecnológicas necesarias en el mercado laboral (Trucano, 2013ab).

Esto demuestra que la correlación entre la variable independiente planteada en cómo los mooc basados en un modelo pedagógico adaptativo genera un efecto en la variable dependiente, dadas las experiencias de aprendizaje que se materializan desde dos aspectos, a saber: primero, respecto a los procesos de aprendizaje monitoreados a partir del desarrollo de las actividades, como condición para avanzar de un nivel a otro. El segundo aspecto se centró en el monitoreo de la permanencia de los participantes, identificando condiciones como baja deserción y alta certificación.

De hecho, el modelo y sus dimensiones permitieron un aprendizaje exitoso y adaptativo, a partir de la metodología del ABP, que genera experiencias de aprendizaje mediante casuísticas propias del contexto laboral de los participantes. Por esto, el modelo planteado estuvo al nivel de experiencias como la desarrollada por Moser-Mercer (2014), quien hizo un estudio con personas pertenecientes al Campo de Refugiados de Dadaab (Kenia) en un MOoc de Coursera.

Moser-Mercer (2014) sugiere que los MOoc en estos contextos precisan de una adaptación de las herramientas para que sean operativos en áreas con baja conectividad. Del mismo modo, se debe desarrollar una didáctica que permita al estudiante interactuar con sujetos en situaciones similares. Por tanto, la dimensión humanitaria de las zonas en conflicto requiere de un diseño, desarrollo y puesta en práctica que respete el Derecho Internacional Humanitario (Moser-Mercer, 2014). Por esto, es claro que sin importar que los participantes tuvieran diferencias en cuanto a género, contextos laborales (rurales o urbanos), niveles educativos, campos de acción laboral, fue posible encontrar contenidos comunes a sus contextos, generando aprendizajes significativos.

Frente a las variables extrañas, centradas en los posibles problemas de conectividad, se puede afirmar que $2 \%$ de los participantes no pudieron finalizar sus mooc debido a problemas de acceso a Internet, situación ajena a los investigadores y, por tanto, a la operacionalización del proyecto. Esto se evidenció en los reportes generados por la plataforma, pues al rastrear los lugares donde no se abordaron los diferentes MOOc, coincidía con la caracterización de dicho contexto. 
Diseño pedagógico adaptativo para el desarrollo de Mooc: una estrategia para el desarrollo de competencias en contextos corporativos

Estos sustentos permiten corroborar la Hi (hipótesis de investigación): el uso de un mooc compuesto por un modelo pedagógico adaptativo favorece los procesos de aprendizaje que aportan a la gestión institucional y al desarrollo de competencias tecnológicas, producto de una guía didáctica con principios, rasgos metodológicos y recursos didácticos flexibles. Sin duda, esta afirmación se hace patente en los resultados obtenidos por cada participante, a partir de la rúbrica de evaluación del curso conocida como "gamificación".

En el proceso de implementación los estudiantes avanzaban de una fase de "reconocimiento y definición" con 90 puntos (al obtener éxito en el desarrollo de sus actividades de aprendizaje, calificadas de manera automática por el sistema) hacia una fase de "apropiación" con otros 90 puntos para certificarse con un total de 180. Es así como los resultados muestran que se certificó el 60\% de los participantes (con 180 puntos cada uno); por tanto, es evidente la sinergia entre el seguimiento a los procesos de aprendizaje, consolidados en el desarrollo adecuado de las actividades, y el monitoreo de la permanencia o nivel de deserción de los usuarios.

De acuerdo con estos resultados se rechaza la Ho (Hipótesis nula) que sustentaba que el uso de un Mooc, compuesto por un modelo pedagógico adaptativo, no favorece los procesos de aprendizaje que aportan a la gestión institucional y al desarrollo de competencias tecnológicas producto de una guía didáctica con principios, rasgos metodológicos y recursos didácticos, pues fueron los componentes del modelo pedagógico los que brindaron recursos para lograr el éxito en el aprendizaje, concretando así las fases del MOOC.

\section{Conclusión}

Gracias a los rastreos realizados se identificaron aspectos que han obstaculizado el acceso de los mooc a determinada población. No obstante, esta experiencia evidenció su viabilidad en el marco de un modelo pedagógico adaptativo, a partir de una metodología que lo operacionalizó, compuesto por una guía didáctica con principios, rasgos metodológicos y recursos didácticos flexibles.

En cuanto a los aspectos analizados en las hipótesis y las variables de esta investigación, tales como: el modelo pedagógico adaptativo, el favorecimiento de los procesos de aprendizaje en el campo de la gestión institucional y el desarrollo de competencias tecnológicas producto de una guía didáctica con principios, rasgos metodológicos y recursos didácticos flexibles, a continuación se presentan las conclusiones y alcances de cada aspecto.

Respecto al modelo pedagógico se puede concluir que ha sido el aprendizaje adaptativo el que dinamiza el modelo, toda vez que las estrategias y los recursos embebidos en el mooc se centraron en el aprendizaje contextual: en esta investigación se materializaron a partir del planteamiento de actividades, que llevaban a los participantes a solucionar problemas de su contexto corporativo. Esto implicó partir del conocimiento práctico que poseían, sin descuidar cierta información sobre la forma de realizarla. Allí era necesario que el usuario acudiera a los componentes de la fase Teorizar del MOoc, un espacio que contenía dichos insumos para lograr resolver el ejercicio.

Este tipo actividades favoreció los procesos de aprendizaje en el campo de la gestión institucional y el desarrollo de competencias tecnológicas, a partir del planteamiento de recursos y estrategias didácticas enmarcadas en situaciones reales en forma de casos, solicitar a los participantes la identificación de problemas y las alternativas de solución. Sin embargo, cuando no se cuenta con la experiencia y conocimiento sobre ello, difícilmente podrá reconocer el problema, y tendrá serios problemas al proponer soluciones viables. Por tal razón, los componentes concentrados en las fases del mooc -para practicar y teorizar-,permitió a los usuarios encontrar aquellos elementos que necesitaba para completar la actividad y aprender. Esto posibilitó su adaptabilidad, pues cada participante recorrió el mooc y usó los recursos a partir de sus necesidades de aprendizaje y sus saberes previos.

En su mayoría, los participantes llegaron al nivel de "apropiación" culminando el 100\% del mooc. Esto implicó obtener calificaciones de 180 puntos, la máxima para llegar a este nivel. Fue evidente el progreso 
en el aprendizaje de los participantes y se pudo monitorear el éxito del mismo a partir del avance de un nivel a otro. Una variable extraña que no se contempló en la investigación fue el poder apreciar mayor motivación por continuar aprendiendo dentro del Mooc, toda vez que la evaluación automática le permitía al usuario ver cuánto aprendía, materializando los avances de su aprendizaje en el progreso de cada nivel dentro del mooc.

Respecto a la guía didáctica con principios, rasgos metodológicos y recursos didácticos flexibles, se puede concluir que resultaron ser valiosas y solventes en el proceso de aprendizaje de los participantes en el mooc. Aunque la población poseía diversos niveles de formación académica, contextos laborales diversos o poca experiencia al aplicar el conocimiento en el contexto corporativo, los recursos brindaron herramientas cognitivas para analizar situaciones con un mayor nivel de complejidad, así como trasladar el conocimiento práctico a una situación real de aprendizaje.

Cabe resaltar que aunque una de las estrategias didácticas en casuísticas no equivale necesariamente a un aprendizaje adaptativo (Jiménez, I., Ortiz, V. 2018), sí constituye un soporte para desarrollar conocimientos y habilidades necesarios para resolver un problema diseñando alternativas de solución. Es aquí donde las TIC jugaron un papel fundamental, como herramienta que permite recrear las diversas situaciones. En este sentido, usar las TIC como un andamiaje didáctico permite emular lo que encontrará en su realidad profesional, donde adapta su experiencia a las casuísticas a las que el usuario del mooc se enfrenta.

Algunos de los logros en el aprendizaje obtenidos por los participantes que se desempeñan en los sectores corporativos, y que responden al objetivo de esta investigación, centrado en "determinar el aporte de los MOoc con base en un modelo pedagógico adaptativo en los procesos de aprendizaje de sus usuarios para la gestión institucional y al desarrollo de competencias tecnológicas en usuarios de contextos corporativos", pueden clasificarse en procesos transversales como:

- Validar sus propios conceptos de aprendizaje producto de sus experiencias en el contexto laboral llevando a la revisión teórica de conceptos embebidos en los MOOc, que les permitió cualificar sus saberes generando trasposiciones de la práctica a la teoría.

- Identificar las actitudes que poseen los participantes para generar procesos de intervención en el sector corporativo reconociendo sus saberes prácticos sobre los temas planteados.

- Comprender la amplitud de términos y conceptos enmarcados su práctica corporativa, revirtiendo en su contexto los conocimientos adquiridos en el mooc.

- Identificar estrategias para resolver problemáticas de contexto en el sector corporativo desarrollando competencias que aportan a la gestión institucional.

Por último, este artículo permite validar el uso de mooc a partir de modelos pedagógicos que robustezcan su práctica. Esto lo evidencia la oferta de más de 4000 cursos mooc en el mundo, donde se ha demostrado la calidad de la enseñanza y del aprendizaje en línea. De hecho, estos cursos conducen a créditos y grados que demuestran su éxito en la certificación, producto de la resolución rigurosa de las actividades de aprendizaje propuestas para este fin.

Las prácticas de literacidad en la escuela han estado históricamente basadas en logros lingüísticos, tanto escritos como orales. En específico, en la evaluación de la capacidad de manejar habilidades discretas como la conciencia fonémica, la ortografía, la puntuación, decodificación y comprensión (Cope y Kalantzis, 2000). En Chile las prácticas de literacidad surgieron históricamente en el siglo XIX como una "garantía del progreso político y económico" (Ponce de León, 2010), para luego pasar a ser un eje central para el desarrollo humano, entendido éste como la capacidad de inserción efectiva de los individuos en la sociedad a nivel económico, social y cívico (OECD, 2010). 


\section{Referencias}

Bates, T. (2012). What's right and what's wrong about Coursera-style moocs?

https://www.tonybates.ca/2012/08/05/whats-right-and-whats-wrong-about-coursera-style-moocs/

Cann, A. (2013). After the gold rush: moocs are augmenting rather than replacing formal educational models. http://blogs.lse.ac.uk/impactofsocialsciences/2013/01/16/after-the-gold-rush/

Daradoumis, T., Bassi, R., Xhafa, F. y Caballé, S. (2013). A review on massive e-learning (MOoc) design, delivery and assessment. Eighth International Conference on P2P, Parallel, Grid, Cloud and Internet Computing. IEEE Computer Society, 208-213. https://doi.org/10.1109/3PGCIC.2013.37

Díaz, F. (2006). Enseñanza situada: vínculo entre la escuela y la vida. McGraw Hill.

Fernández, S. (1993). Educación adaptativa como respuesta a la diversidad. Signos. Teoría y práctica de la educación, 8(9), pp. 128-139.

Gaebel, M. (2014). Moocs Massive Open Online Courses. https://www.oerknowledgecloud.org/record720

Hollands, F. y Tirthali, D. (2014). moocs: expectations and reality. Columbia University, Center for Benefit-Cost Studies of Education, Teachers College. https://www.cbcse.org/s/MOOCs Expectations and Reality.pdf

Kolowich, S. (2013). American Council on Education recommends 5 moocs for credit. The chronicle of higher education. https://www.chronicle.com/article/American-Council-on-Education/137155/

Liyanagunawardena, T., Williams, S. y Adams, A. (2013). The Impact and Reach of Moocs: a developing countries' perspective. elearning Papers, 33. http://centaur.reading.ac.uk/32452/

Mak, S., Williams, R. y Mackness, J. (2010). Blogs and forums as communication and learning tools in a mooc. En L. Dirckinck-Holmfeld, V. Hodgson, C. Jones, M. de Laat, D. McConnell y T. Ryberg (Eds.) Proceedings of the 7th International Conference on Networked Learning 2010 (pp. 275-284). University of Lancaster.

Ministerio de Tecnologías de la Información y las Comunicaciones (2015a). Mooc: adopción del protocolo IPV6" y "Mejores prácticas en la gestión y seguridad de tI". Colombia.

Ministerio de Tecnologías de la Información y las Comunicaciones (2015b). Informe Final Convenio 475 de 2015: estrategia de sistematización de la experiencia desarrollada para los MOoc de Mintic y RENATA, en los sectores y entidades del Estado. Colombia.

Moser-Mercer, B. (2014). moocs in fragile contexts. European moocs Stakeholders Summit. https://www.unige.ch/inzone/Files/9514/8965/4778/MOOCsinFragileContexts.pdf

Palacios, Coll y Marchesi (2015) Desarrollo psicológico y educación. Alianza.

Parr, C. (2013). Not staying the course. https://www.insidehighered.com/news/2013/05/10/new-study-lowmooc-completion-rates

Postic, M. (2000). La relación educativa: factores institucionales, sociológicos y culturales. Narcea.

Rojas, R. (1989). Investigación social teoría y praxis. Plaza y Valdez. 
Snow, R. (1997). Aptitudes and symbol systems in adaptive classroom teaching. Phi Delta Kappan, 78(5). 354-360.

Trucano, M. (2013a). moocs in Africa. https://blogs.worldbank.org/edutech/moocs-in-africa

UNESCO. (2013). Introduction to moocs: Avalanche, illusion or augmentation?

https://iite.unesco.org/publications/3214722/

Universities United Kingdom (2013). Massive open online courses: higher education's digital moment? https://www.universitiesuk.ac.uk/policy-and-analysis/reports/Pages/massive-open-online-courses.aspx

Warnock, M. (1978). Informe sobre necesidades educativas especiales. Siglo Cero. 